\title{
Regression Estimators in Ranked Set, Median Ranked Set and Neoteric Ranked Set Sampling
}

\author{
Nursel Koyuncu \\ Department of Statistics \\ Hacettepe University, Turkey \\ nkoyuncu@ hacettepe.edu.tr
}

\begin{abstract}
This paper proposes new regression estimators in median ranked set and neoteric ranked set sampling using one and two auxiliary variables. The proposed estimators have large gain in presicion compared to the classical ranked set sampling (RSS) design. A simulation study is designed to see the performance of suggested estimators. A real data set example is also used. In this data set, we have examined a rare endemic annual plant species which is grown in Turkey. We have found that suggested estimators are highly efficient that existing estimators.
\end{abstract}

Keywords: Median Ranked Set Sampling, Neoteric Ranked Set Sampling, Regression Estimator, Efficiency.

\section{Introduction}

Ranked set sampling (RSS) is first proposed by McIntyre (1952). He discussed this sampling design is often more efficient than simple random sampling of the same size. Many authors in sampling literature have modified this samplign design to get more efficiency such as Al-Saleh and Al-Omari (2002), Jemain and Al-Omari (2006), Jemain et al. (2007), Zamanzade and Al-Omari (2016). Moreover Al-Omari (2012), Koyuncu (2015, 2016), Zamanzade and Vock (2015) have utilized information of auxiliary variable in various median ranked set sampling. Recently, Zamanzade and Al-Omari (2016) have introduced neoteric ranked set sampling (NRSS). In this study we have proposed regression estimators in median ranked set sampling (MRSS) and neoteric ranked set sampling. To compare the efficiency we have conducted simulation study with simulated and real data sets.

\section{Ranked Set Sampling}

Ranked Set Sampling (RSS) design can be described as follows:

1. Select a simple random sample of size $n^{2}$ units from the target finite population and divide them into $n$ samples each of size $n$.

2. Rank the units within each sample in increasing magnitude by using personal judgment, eye inspection or based on a concomitant variable.

3. Select the $i$ th ranked unit from the $i$ th $(i=1,2, \ldots, n)$ sample

4. Repeat steps 1 through $3 m$ times if needed to obtain a RSS of size $\mathrm{N}=n m$. 
Let $\left(X_{1}, Z_{1}, Y_{1}\right),\left(X_{2}, Z_{2}, Y_{2}\right), \ldots,\left(X_{n}, Z_{n}, Y_{n}\right)$ be a simple random sample of size $n$, then the measured ranked set sampling units are denoted by $\left\{\left(X_{(i) j}, Z_{(i) j}, Y_{[i] j}\right), i=1, \ldots, n, j=1, \ldots, m\right\}$ where $\left(X_{(i) j}, Z_{(i) j}, Y_{[i] j}\right)$ is the ith ranked unit from the jth cycle of two auxiliary variables and study variable respectively, ( ) and [ ] indicate that the ranking of $X$ and $\mathrm{Z}$ is perfect and ranking of $Y$ has errors. In this paper we prefer to rank the units one of the most correlated auxiliary variables with study variable. The sample means of variables can be defined as in RSS:

$$
\bar{y}_{R S S}=\frac{1}{n m} \sum_{j=1}^{m} \sum_{i=1}^{n} Y_{[i] j}, \quad \bar{x}_{R S S}=\frac{1}{n m} \sum_{j=1}^{m} \sum_{i=1}^{n} X_{(i) j}, \bar{z}_{R S S}=\frac{1}{n m} \sum_{j=1}^{m} \sum_{i=1}^{n} Z_{(i) j}
$$

We introduce regression estimators using one and two auxiliary information, respectively, as:

$$
\begin{aligned}
& \bar{y}_{\text {Re } 1_{1} R S S}=\bar{y}_{R S S}+b_{R S S 1}\left(\mu_{x}-\bar{x}_{R S S}\right) \\
& \bar{y}_{\text {Re } 2_{2} R S S}=\bar{y}_{R S S}+b_{R S S 1}\left(\mu_{x}-\bar{x}_{R S S}\right)+b_{R S S 2}\left(\mu_{z}-\bar{z}_{R S S}\right)
\end{aligned}
$$

\section{Median Ranked Set Sampling}

Al-Omari (2012) has introduced (MRSS) as follows:

1. Select $n$ random samples each of size $n$ bivariate units from the finite population of interest.

2. The units within each sample are ranked by visual inspection or any other cost free method with respect to a variable of interest.

3. If $n$ is odd, select the $\left(\frac{n+1}{2}\right)$ th-smallest ranked unit $X$ together with the associated $Y$ from each set, i.e., the median of each set. If $n$ is even, from the first $\frac{n}{2}$ sets select the $\left(\frac{n}{2}\right)$ th ranked unit $\mathrm{X}$ together with the associated $\mathrm{Y}$ and from the other $\frac{n}{2}$ sets select the $\left(\frac{n+2}{2}\right)$ th ranked unit $X$ together with the associated $Y$.

4. The whole process can be repeated $m$ times if needed to obtain a sample of size $n m$ units.

Let $\left(X_{i(1)}, \mathrm{Z}_{i(1)}, Y_{i[1]}\right),\left(X_{i(2)}, \mathrm{Z}_{i(2)}, Y_{i[2]}\right), \ldots,\left(X_{i(n)}, \mathrm{Z}_{i(n)}, Y_{i[n]}\right)$ be the order statistics of $X_{i 1}, X_{i 2}, \ldots, X_{i n}, Z_{i 1}, Z_{i 2}, \ldots, Z_{i n}$ and the judgment order of $Y_{i 1}, Y_{i 2}, \ldots, Y_{i n}(i=1,2, \ldots, n)$, For odd and even sample sizes the units measured using MRSS are denoted by MRSSO 
and MRSSE, respectively. For odd sample size let $\left(X_{1\left(\frac{n+1}{2}\right)}, Z_{1\left(\frac{n+1}{2}\right)}, Y_{1\left[\frac{n+1}{2}\right]}\right)$, $\left(X_{2\left(\frac{n+1}{2}\right)}, \mathrm{Z}_{2\left(\frac{n+1}{2}\right)}, Y_{2\left[\frac{n+1}{2}\right]}\right), \ldots,\left(X_{n\left(\frac{n+1}{2}\right)}, \mathrm{Z}_{n\left(\frac{n+1}{2}\right)}, Y_{n\left[\frac{n+1}{2}\right]}\right)$ denote the observed units by MRSSO.

$$
\bar{x}_{\text {MRSSO }}=\frac{1}{n} \sum_{i=1}^{n} X_{i\left(\frac{n+1}{2}\right)}, \bar{z}_{\text {MRSSO }}=\frac{1}{n} \sum_{i=1}^{n} Z_{i\left(\frac{n+1}{2}\right)} \text { and } \bar{y}_{\text {MRSSO }}=\frac{1}{n} \sum_{i=1}^{n} Y_{i\left[\frac{n+1}{2}\right]}
$$

be the sample mean of $X, \mathrm{Z}$ and $Y$ respectively in (MRSS).

For even sample size let $\left(X_{1\left(\frac{n}{2}\right)}, \mathrm{Z}_{1\left(\frac{n}{2}\right)}, Y_{1\left[\frac{n}{2}\right]}\right),\left(X_{2\left(\frac{n}{2}\right)}, \mathrm{Z}_{2\left(\frac{n}{2}\right)}, Y_{2\left[\frac{n}{2}\right]}\right), \ldots,\left(X_{\frac{n}{2}\left(\frac{n}{2}\right)}, \mathrm{Z}_{\frac{n}{2}\left(\frac{n}{2}\right)}, Y_{\frac{n}{2}\left[\frac{n}{2}\right]}\right)$ $,\left(X_{\frac{n+2}{2}\left(\frac{n+2}{2}\right)}, \mathrm{Z}_{\frac{n+2}{2}\left(\frac{n+2}{2}\right)}, Y_{\frac{n+2}{2}\left[\frac{n+2}{2}\right]}\right)\left(X_{\frac{n+4}{2}\left(\frac{n+2}{2}\right)}, \mathrm{Z}_{\frac{n+4}{2}\left(\frac{n+2}{2}\right)}, Y_{\frac{n+4}{2}\left[\frac{n+2}{2}\right]}\right),\left(X_{n\left(\frac{n+2}{2}\right)}, \mathrm{Z}_{n\left(\frac{n+2}{2}\right)}, Y_{n\left[\frac{n+2}{2}\right]}\right)$ denote the observed units by MRSSE.

$$
\begin{aligned}
& \bar{x}_{\text {MRSSE }}=\frac{1}{n}\left(\sum_{i=1}^{\frac{n}{2}} X_{i\left(\frac{n}{2}\right)}+\sum_{i=\frac{n+2}{2}}^{n} X_{i\left(\frac{n+2}{2}\right)}\right), \bar{z}_{\text {MRSSE }}=\frac{1}{n}\left(\sum_{i=1}^{\frac{n}{2}} Z_{i\left(\frac{n}{2}\right)}+\sum_{i=\frac{n+2}{2}}^{n} Z_{i\left(\frac{n+2}{2}\right)}\right) \text { and } \\
& \bar{y}_{\text {MRSSE }}=\frac{1}{n}\left(\sum_{i=1}^{\frac{n}{2}} Y_{i\left[\frac{n}{2}\right]}+\sum_{i=\frac{n+2}{2}}^{n} Y_{i\left[\frac{n+2}{2}\right]}\right)
\end{aligned}
$$

be the sample mean of $X, \mathrm{Z}$ and $Y$ respectively.

In MRSS we can re-write estimators in defined in section two as given by, respectively

$$
\begin{aligned}
& \bar{y}_{\text {Re } 1_{1} \_ \text {MRSS }}=\bar{y}_{\text {MRSS }}+b_{M R S S 1}\left(\mu_{x}-\bar{x}_{M R S S}\right) \\
& \bar{y}_{\text {Re } 2_{2} \_M R S S}=\bar{y}_{M R S S}+b_{\text {MRSS1 }}\left(\mu_{x}-\bar{x}_{M R S S}\right)+b_{\text {MRSS } 2}\left(\mu_{z}-\bar{z}_{M R S S}\right)
\end{aligned}
$$

\section{Neoteric Ranked Set Sampling}

Zamanzade and Al-Omari (2016) have defined a new neoteric ranked set sampling (NRSS). The NRSS scheme can be described as follows:

1. Select a simple random sample of size $n^{2}$ units from the target finite population.

2. Ranked the $\mathrm{n}^{2}$ selected units in an increasing magnitude based on a concomitant variable, personal judgment or any inexpensive method. 


\section{Nursel Koyuncu}

3. If $\mathrm{n}$ is an odd, then select the $\left[\frac{\mathrm{n}+1}{2}+(\mathrm{i}-1) \mathrm{n}\right]$ th ranked unit for $\mathrm{i}=1, \ldots, \mathrm{n}$. If $\mathrm{n}$ is an even, then select the $[\ell+(i-1) n]$ th ranked unit, where $\ell=\frac{n}{2}$ if $\mathrm{i}$ is an even and $\ell=\frac{n+2}{2}$ if $i$ is an odd for $i=1, \ldots, n$.

4. Repeat steps 1 through $3 m$ times if needed to obtain a NRSS of size $\mathrm{N}=n m$.

Let $\left(X_{1 j}, Z_{1 j}, Y_{1 j}\right),\left(X_{2 j}, Z_{2 j}, Y_{2 j}\right), \ldots,\left(X_{n^{2} j}, Z_{n^{2} j}, Y_{n^{2} j}\right)$ be $n^{2}$ simple random units selected from the population of interest and let $\left(X_{(1) \mathrm{j}}, Z_{(1) \mathrm{j}}, Y_{[1] \mathrm{j}}\right),\left(\mathrm{X}_{(2) \mathrm{j}}, \mathrm{Z}_{(2) \mathrm{j}}, \mathrm{Y}_{[2] \mathrm{j}}\right), \ldots$,

$$
\begin{gathered}
\left(\mathrm{X}_{\left(\mathrm{n}^{2}\right) \mathrm{j}}, \mathrm{Z}_{\left(\mathrm{n}^{2}\right) \mathrm{j}}, \mathrm{Y}_{\left[\mathrm{n}^{2}\right] \mathrm{j}}\right) \text { be } \quad \text { order } \\
\left(\mathrm{X}_{1 \mathrm{j}}, \mathrm{Z}_{1 \mathrm{j}}, \mathrm{Y}_{1 \mathrm{j}}\right),\left(\mathrm{X}_{2 \mathrm{j}}, \mathrm{Z}_{2 \mathrm{j}}, \mathrm{Y}_{2 \mathrm{j}}\right), \ldots,\left(\mathrm{X}_{\mathrm{n}^{2} \mathrm{j}}, \mathrm{Z}_{\mathrm{n}^{2} \mathrm{j}}, \mathrm{Y}_{\mathrm{n}^{2} \mathrm{j}}\right) \text { for } \mathrm{j}=1, \ldots, \mathrm{m} . \\
\bar{y}_{N R S S}=\frac{1}{n m} \sum_{j=1}^{m} \sum_{i=1}^{n} Y_{[(i-1) n+\ell] j}, \bar{x}_{N R S S}=\frac{1}{n m} \sum_{j=1}^{m} \sum_{i=1}^{n} X_{((i-1) n+\ell) j} \\
\bar{z}_{N R S S}=\frac{1}{n m} \sum_{j=1}^{m} \sum_{i=1}^{n} Z_{((i-1) n+\ell) j}
\end{gathered}
$$

be the sample means of study and auxiliary variables in (NRSS). And now we can define regression estimators in (NRSS) as follows:

$$
\begin{aligned}
& \bar{y}_{\text {Re } 21 \_\mathrm{N} R S S}=\bar{y}_{N R S S}+b_{N R S S 1}\left(\mu_{x}-\bar{x}_{N R S S}\right) \\
& \bar{y}_{\text {Re } 2_{2} \_\mathrm{N} R S S}=\bar{y}_{N R S S}+b_{N R S S 1}\left(\mu_{x}-\bar{x}_{N R S S}\right)+b_{N R S S 2}\left(\mu_{z}-\bar{z}_{N R S S}\right)
\end{aligned}
$$

\section{Simulation Study}

In this section, we conducted a simulation study to investigate the properties of proposed estimators. In the simulation study, we consider finite populations of size $N=10000$ generated from a bivariate normal distribution. The samples were generated from a bivariate normal distribution using mvrnorm function in $\mathrm{R}$ programme. In the simulation, we considered $\mu_{x}=2, \mu_{z}=3, \mu_{y}=4, \rho_{x y}=0.80, \rho_{z y}=0.75$. We have computed mean square errors (MSEs) and percent relative efficiencies (PREs) of estimators with respect to $\bar{y}_{R S S}$ for $n=3,4,5,6,7$ on the basis of 60.000 replications and displayed in Table1. From the Table1 we can say that regression estimators are more efficient in (NRSS) and (MRSS) compared to (RSS). We can also see that when the sample size is odd, the regression estimators in (NRSS) are more efficient than regression estimators in (MRSS). 


\section{Real Data Set}

In this data set, we have examined a rare endemic annual plant species which is grown in Ankara-Turkey. 896 seeds of endemic plant's weight, height and papus are measured. Using this data set as a population by setting height as study variable (Y) and weight (X) and papus $(Z)$ as auxiliary variables. We have selected 10000 sample under median ranked set and neoteric ranked set sampling design. From each sample we have estimated the height of plant with suggested estimators and calculated mean square error. According to mean square error value we have decided that which sampling plan is suitable for this data set. Descriptive statistics of data set: $\mu_{x}=1.29, \mu_{z}=1.97$, $\mu_{y}=2.99, \rho_{x y}=0.834, \rho_{z y}=0.703$. The result of real data set is summarized in Table 2 .

\section{Conclusion}

In this study, we have introduced regression estimators in median and neoteric ranked set sampling designs which are recently introduced by Al-Omari (2012) and Zamanzade and Al-Omari (2016). We have compared the efficiency of our suggested estimators with AlOmari (2012) and Zamanzade and Al-Omari (2016) estimators. We have found that our suggested regression estimators are highly efficient than existing estimators.

\section{References}

1. Al-Omari, A.I. (2012). Ratio estimation of the population mean using auxiliary information in simple random sampling and median ranked set sampling. Statistics and Probability Letters, 82, 1883-1890.

2. Al-Saleh, M.F. and Al-Omari, A.I. (2002). Multistage ranked set sampling. Journal of Statistical Planning and Inference, 102, 273-286.

3. Jemain, A.A. and Al-Omari, A.I. (2006). Multistage median ranked set samples for estimating the population mean. Pakistan Journal of Statistics, 22, 195-207

4. Jemain, A.A., Al-Omari, A.I. and Ibrahim, K. (2007). Multistage extreme ranked set sampling for estimating the population mean. Journal of Statistical Theory and Application, 6(4), 456-471.

5. Koyuncu N. (2016). New difference-cum-ratio and exponential type estimators in median ranked set sampling. Hacettepe Journal of Mathematics and Statistics, 45, 1, 207-225.

6. Koyuncu N. (2015). Ratio estimation of the population mean in extreme ranked set and double robust extreme ranked set sampling. International Journal of Agricultural and Statistical Sciences, 11(1), 21-28.

7. McIntyre, G.A. (1952). A method for unbiased selective sampling using ranked sets. Australian Journal of Agricultural Research, 3, 385-390.

8. Zamanzade, E. and Al-Omari, A.I. (2016). New ranked set sampling for estimating the population mean and variance. Hacettepe Journal of Mathematics and Statistics, 45(6), 1891-1905.

9. Zamanzade, E. and Vock, M. (2015). Variance estimation in ranked set sampling using concomitant variable. Statistics \& Probability Letters, 105, 1-5. 


\section{Nursel Koyuncu}

Table 1: MSE and Efficiency of Estimators

\begin{tabular}{|c|c|c|c|c|c|c|c|c|c|c|}
\hline \multirow[b]{2}{*}{ Estimator } & \multicolumn{2}{|c|}{$n=3$} & \multicolumn{2}{|c|}{$n=4$} & \multicolumn{2}{|c|}{$n=5$} & \multicolumn{2}{|c|}{$n=6$} & \multicolumn{2}{|c|}{$n=7$} \\
\hline & MSE & Efficiency & MSE & Efficiency & MSE & Efficiency & MSE & Efficiency & MSE & Efficiency \\
\hline $\bar{y}_{R S S}$ & 0,2299 & 100,00 & 0,1567 & 100,00 & 0,1188 & 100,00 & 0,0941 & 100,00 & 0,0771 & 100,00 \\
\hline $\bar{y}_{\operatorname{Re} g 1 \_R S S}$ & 0,1210 & 190,08 & 0,0907 & 172,79 & 0,0732 & 162,29 & 0,0608 & 154,81 & 0,0518 & 148,69 \\
\hline $\bar{y}_{\operatorname{Re} g 2_{-} R S S}$ & 0,0440 & 522,78 & 0,0317 & 493,83 & 0,0248 & 478,37 & 0,0202 & 464,56 & 0,0172 & 449,33 \\
\hline $\bar{y}_{N R S S}$ & 0,1979 & 116,15 & 0,1419 & 110,47 & 0,0989 & 120,17 & 0,0811 & 115,97 & 0,0654 & 117,86 \\
\hline $\bar{y}_{\operatorname{Re} g 1 \_\mathrm{N} R S S}$ & 0,1206 & 190,55 & 0,0907 & 172,78 & 0,0716 & 166,05 & 0,0603 & 155,95 & 0,0522 & 147,80 \\
\hline $\bar{y}_{\operatorname{Re} g 2 \_\mathrm{N} R S S}$ & 0,0415 & $553,75^{*}$ & 0,0302 & 519,61 & 0,0235 & $506,54 *$ & 0,0196 & 479,05 & 0,0163 & 472,10 * \\
\hline $\bar{y}_{M R S S}$ & 0,2121 & 108,39 & 0,0910 & 172,25 & 0,1079 & 110,15 & 0,0526 & 178,95 & 0,0693 & 111,31 \\
\hline $\bar{y}_{\operatorname{Re} g 1 \_\mathrm{M} R S S}$ & 0,1207 & 190,42 & 0,0450 & 348,48 & 0,0720 & 164,96 & 0,0302 & 311,41 & 0,0506 & 152,30 \\
\hline $\bar{y}_{\operatorname{Re} g 2 \_\mathrm{M} R S S}$ & 0,0427 & 538,06 & 0,0168 & $934,85^{*}$ & 0,0238 & 498,88 & 0,0106 & $883,92 *$ & 0,0166 & 464,62 \\
\hline
\end{tabular}

\section{*Represent most efficient estimator}

Table 2: MSE and Efficiency of Estimators

\begin{tabular}{|c|c|c|c|c|c|c|c|c|c|c|}
\hline & \multicolumn{2}{|c|}{$n=3$} & \multicolumn{2}{|c|}{$n=4$} & \multicolumn{2}{|c|}{$n=5$} & \multicolumn{2}{|c|}{$n=6$} & \multicolumn{2}{|c|}{$n=7$} \\
\hline Estimator & MSE & Efficiency & MSE & Efficiency & MSE & Efficiency & MSE & Efficiency & MSE & Efficiency \\
\hline $\bar{y}_{R S S}$ & 0,1068 & 100,00 & 0,0711 & 100,00 & 0,0517 & 100,00 & 0,0404 & 100,00 & 0,0315 & 100,00 \\
\hline $\bar{y}_{\operatorname{Re} g 1 \_R S S}$ & 0,0485 & 220,17 & 0,0370 & 192,28 & 0,0293 & 176,41 & 0,0249 & 161,95 & 0,0200 & 157,44 \\
\hline $\bar{y}_{\operatorname{Re} g 2_{2} R S S}$ & 0,0656 & 162,69 & 0,0477 & 148,90 & 0,0376 & 137,46 & 0,0316 & 127,87 & 0,0261 & 120,31 \\
\hline $\bar{y}_{N R S S}$ & 0,0768 & 138,98 & 0,0479 & 148,53 & 0,0318 & 162,86 & 0,0235 & 171,92 & 0,0182 & 172,45 \\
\hline $\bar{y}_{\operatorname{Re} g 1 \_\mathrm{N} R S S}$ & 0,0279 & 382,42 & 0,0194 & 365,58 & 0,0148 & 348,72 & 0,0118 & 342,49 & 0,0095 & 331,30 \\
\hline $\bar{y}_{\operatorname{Re} g 2 \_N R S S}$ & 0,0479 & 222,91 & 0,0353 & 201,23 & 0,0264 & 195,80 & 0,0216 & 186,62 & 0,0183 & 171,74 \\
\hline $\bar{y}_{M R S S}$ & 0,1416 & 75,41 & 0,0696 & 102,11 & 0,0893 & 57,88 & 0,0615 & 65,69 & 0,0822 & 38,27 \\
\hline $\bar{y}_{\operatorname{Reg} 1 \_M R S S}$ & 0,0396 & 269,70 & 0,0164 & 434,29 & 0,0278 & 186,29 & 0,0159 & 254,63 & 0,0256 & 122,94 \\
\hline $\bar{y}_{\operatorname{Reg} 2 \_\mathrm{M} R S S}$ & 0,0427 & 249,96 & 0,0171 & 414,66 & 0,0220 & 234,76 & 0,0098 & 414,22 & 0,0145 & 217,02 \\
\hline
\end{tabular}

\section{*Represent most efficient estimator}

